IMECE2005-81715

\title{
INTEGRATION OF SUB-WAVELENGTH NANOFLUIDICS WITH PHOTONIC CRYSTALS
}

\author{
David Erickson ${ }^{1,2}$, Teresa Emery ${ }^{1}$, Troy Rockwood ${ }^{1}$, Axel Scherer $^{1}$ and Demetri Psaltis ${ }^{1}$ \\ 1 Center for Optofluidic Integration, California Institute of Technology, Pasadena, California, 91125 \\ 2 Department of Mechanical and Aerospace Engineering, Cornell University, Ithaca, New York, 14853 \\ de54@cornell.edu.
}

\begin{abstract}
"Optofluidics" represents the marriage of optics, optoelectronics and nanophotonics with fluidics. Such integration represents a new approach for dynamic manipulation of optical properties at length scales both greater than and smaller than the wavelength of light with applications ranging from reconfigurable photonic circuits to fluidically adaptable optics to high sensitivity bio-detection currently under development. The capabilities in terms of fluidic control, mixing, miniaturization and optical property tuning afforded by micro-, nano-fluidics combined with soft lithography based fabrication provides an ideal platform upon which to build such devices. Here we present our technique for integrating soft lithography based nanofluidics with e-beam lithography defined silicon-on-insulator photonic crystals. We demonstrate nanofluidic addressability of single, sub-wavelength, defects within the planar photonic crystal and the dynamic tuning of the guided mode. In this paper we focus on the fabrication, integration and experimental details of this work.
\end{abstract}

\subsection{INTRODUCTION}

Photonic crystals [1] are attractive for controlling optical propagation by introducing pre-engineered defects into an otherwise regular lattice to create spectral filters, tight bend waveguides, resonant cavities [2] and highly efficient lasers [3]. At present techniques for local refractive index modulation in photonic structures is limited to the exploitation of relatively weak non-linear material properties $[4,5]$, where $\Delta \mathrm{n} / \mathrm{n}$ (where $\mathrm{n}$ is the index of refraction) on the order of $10^{-3}$ or lower, and thus requiring either long interaction lengths, high operational power, or the incorporation of resonant elements to enhance the effect. Techniques such as mechanical deformation [6], thermooptics [7], liquid crystal infusion [8], liquid-fluid infusion $[9,11]$ and others $[12,15]$ offer much higher effective
$\Delta \mathrm{n} / \mathrm{n}$. At present, however, these methods rely on globally modifying the refractive index of the entire device. Thus whereas local tunability over small interaction lengths requires the high $\Delta \mathrm{n} / \mathrm{n}$ afforded by these global approaches, the ability to perform such manipulations with the sub-micron scale precision required for advanced photonic devices remains elusive. The development of such a technique could enable the creation of a new class of ultra-compact adaptable photonic circuits.

Nanofluidics provides a solution that enables both localized control and high refractive index modulation. By using modern nanofabrication techniques fluidic networks can be built which confine flows on scales much smaller than the wavelength of the transmitted light and enable the direct infusement and exchange of liquids with interesting optical properties (e.g. varying refractive index, gain and non-linearity) directly into the nanophotonic structure. As a first step in the development of two dimensional reconfigurable photonic circuits, here we demonstrate the nanofluidic addressing of a single defect row of holes within a two dimensional photonic crystal. We begin by detailing our fabrication and integration procedure which is based around a three-level integration technique consisting of a bottom SOI photonic layer, a PDMS nanofluidic structure which is used to directly infuse liquids into the targeted region of the photonic structure and a "microfluidics engine" which serves to perform all the major fluidic manipulations (e.g. mixing and dispensing) at the micron scale and fluidically couple the results into the nanofluidic system. The details of the experimental apparatus and operation will be described and initial experimental results demonstrating the tuning of the guided mode through the fluidically modulated defect in the photonic crystal will be presented. 


\subsection{NANOSCALE OPTOFLUIDIC INTEGRATION}

As mentioned above the integration of fluidics and photonics at the 100 nanometer scale represents a significant challenge in terms of fabrication, alignment and operation. Due to the extreme difficulty in constructing a single structure which contains both the photonic and fluidic components, the general approach used here was to take advantage of standard photonic and fluidic processing (fabricating the two components separately) and then develop a technique for interfacing the two. In this section will first give a brief and general overview of micro- and nano-fabrication techniques and materials in the context of which are most suitable for integration with nanophotonic structures. We will then describe in greater detail the specifics of the fabrication procedure and general integration philosophy used here including it's advantages and limitations.

\subsection{Fabrication Procedure for SOI Photonic Crystals}

The e-beam lithography and dry etching fabrication procedures for creating the SOI photonic crystals used are well established and well described in the literature (e.g. [16]). For optofluidic integration we modify the procedure outlined in Loncar et al. [16] only slightly in that we defined the pattern in a negative flowable oxide resist which is left on after processing to enhance bonding with the fluidic layer. Additionally we do not etch the underside insulator layer in favor of using it to enhance the mechanical stability of the structure. In general, "flow through" photonic structures (such as membrane suspended photonic crystals) require much more complicated fluidic confinement schemes on both sides of the device and therefore well type structures are typically preferred. Figure 1 shows a sample photonic crystal used here prior to integration with the fluidics.

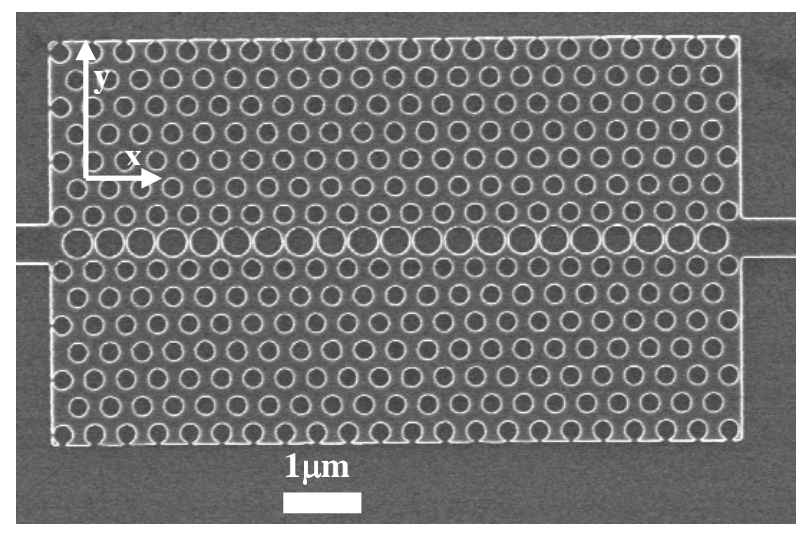

Figure 1: Silicon-on-Insulator Photonic Crystal. The lattice constant and hold radius of the crystal are $434 \mathrm{~nm}$ and $140 \mathrm{~nm}$ respectively and the height is $207 \mathrm{~nm}$ out of the page. Larger holes in the center are a design-time defect done in order to introduce a guided mode into band gap of the crystal. The larger holes are those which are to be targeted fluidically.
2.2 Overview of micro- and nano- fluidic fabrication procedures

At the onset of modern microfluidics in the early 1990's glasses, quartz and silicon were the materials of choice for microfabrication [17-19], primarily due to the well established wet etching and lithography based micromachining techniques developed by the electronics and semiconductor industries [2021]. When defined using e-beam lithography or similar high resolution resist patterning techniques and etched using RIE or equivalent techniques resolutions on the orders required here can be obtained relatively easily. While such structures have the advantage of having a well defined, rigid geometry, optofluidic integration requires the fluidic system to travel over not only the photonic structure but also the coupling waveguides and other protruding optical structures on the base SOI substrate and thus fluidic sealing between the two rigid structures becomes difficult.

The alternative approach is to use some of the more recently developed techniques for building fluidic systems from lowcost polymeric materials such as poly(dimethsiloxane) (PDMS) [22-23], poly(methylmethacrylate) (PMMA) [24-25] and others (see Becker and Locascio [26] and deMello [27] for comprehensive reviews). In general the primary attractiveness of these materials is that they tend to involve simpler and significantly less expensive manufacturing techniques (e.g. casting, injection and replica molding, or hot embossing). Of these materials and techniques, PDMS processing using the soft lithography process developed by Whitesides' group at Harvard University [23, 28, 29] is the most suitable as is electrometric nature facilitates sealing over rigid surfaces. The Quake group, then at Caltech, demonstrated how a reasonably simple two layer structure constructed using this processing can be scaled to create highly integrated devices with 1000's of valves per $\mathrm{cm}^{2}$ (see Thorsen et al. [30] for details). This same feature also complicates the fabrication of sub-micron channels as it deformable nature can to lead to channel collapse, though in general such problems can be avoided by manipulating the linker:base ratios and through careful processing. As a result multi-layer soft-lithography was selected as the fluidic fabrication technique for this work. In addition to manufacturing simplicity, building the fluidic layer separately from the optical layer in a soft polymer such as PDMS or RTV has the additional advantage of being air permeable which facilitates the initial wetting of the system.

\subsection{Multilevel Optofluidic Integration}

From an operational perspective addressing individual features within a photonic crystal necessitates the use of nanoscale fluidics, however the complex fluidic switching, dispensing and mixing required to operate such devices becomes difficult at those length scales due to the $\mathrm{D}^{2}$ dependence of flow velocity on channel size (where $\mathrm{D}$ is the characteristic channel diameter) and $1 / \mathrm{L}^{2}$ dependence of the diffusion time (where $\mathrm{L}$ is the characteristic length scale for diffusion). To avoid this 
difficulty we developed a technique for "multilevel optofluidic integration” as described below.

The first level of integration is the nanophotonic level which comprises of the device or devices to be fluidically targeted, defined on the base substrate which also provides support for the fluidics. In this case we use an array of 30 identical photonic crystal structures defined in SOI, each with a triangular lattice of holes constant $\mathrm{a}=434 \mathrm{~nm}$, hole radius $\mathrm{r}=$ $140 \mathrm{~nm}$ and height $\mathrm{h}=207 \mathrm{~nm}$. In the experiments presented here we increase the radius of the holes within the central row of the photonic crystal (which is to be targeted fluidically) to 203nm in order to introduce a reduced index guided mode into the band gap created by the otherwise regular crystal lattice. This geometry was selected on the basis of a series of numerical experiments as having transmission properties that were most sensitive to changes in index of refraction within the targeted region. Details on the calculations performed to reach this conclusion are outlined in Section 4.

The second level of integration is the nanofluidic level which enables direct fluidic coupling into the photonic structure. In this case the nanofluidic system consists of an array of 350nm wide channels spaced with $5 \mu \mathrm{m}$ period. Using similar e-beam and dry etch processing to that done for the nanophotonic structure, we hard define the nanofluidic layout in positive relieve into a separate SOI substrate (we discuss the use SOI to define the nanofluidics here, though we have also used standard silicon substrates). The relief pattern used here was approximately 200nm tall coinciding with the thickness of the silicon layer.

The purpose of the nanofluidics level is to provide fluidic coupling between the photonic structure and the upper level of integration or "microfluidics engine" as described previously. To integrate the microscale fluidics we define the pattern, again in positive relief, on the same nanofluidic substrate using the following technique. After acetone and isopropyl alcohol cleaning and treating the nanofluidic substrate with an hexamethyldisilazane (HDMS) based photoresist adhesion agent, SPR 220-7 (Shipley) photo resist was spun on to a thickness of approximately $10 \mu \mathrm{m}$. The mask containing the fluidic patterns was then aligned such that the predefined nanochannels overlapped with the microfluidic pattern to ensure efficient coupling between the two structures. The photoresist was then exposed, soft baked and developed using the procedure recommended by the manufacturer. A hard bake step at $110^{\circ} \mathrm{C}$ for at least $30 \mathrm{~min}$ was then done to harden the photoresist and enhance the adhesion. A 20-30 $\mu$ m thick film of 10:1 (base:linker) RTV is then spun on the master and allowed to cure for 30 minutes at $80^{\circ} \mathrm{C}$.
E-beam resist
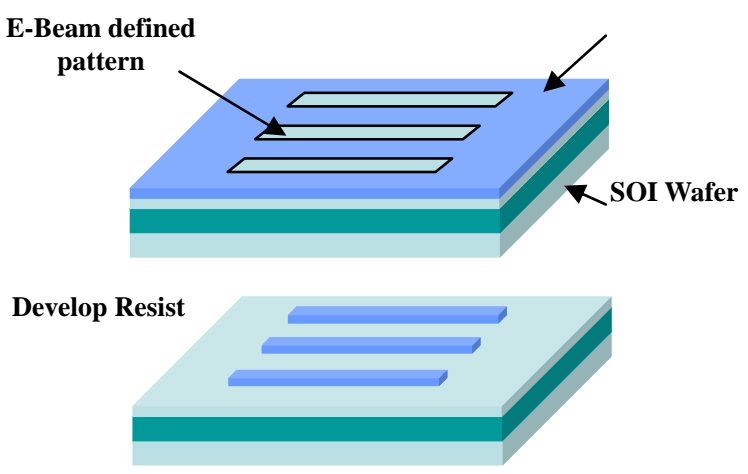

Dry etch to define nano-fluidics

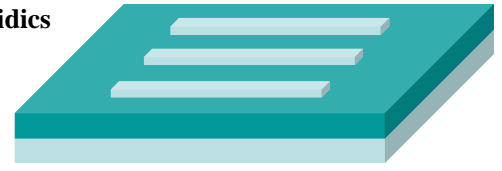

Photolithography to add microfluidics

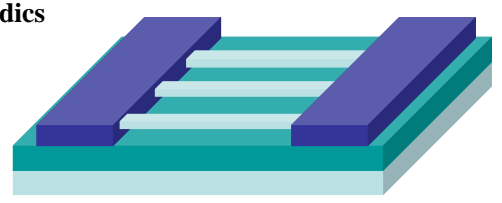

Cast and cure PDMS

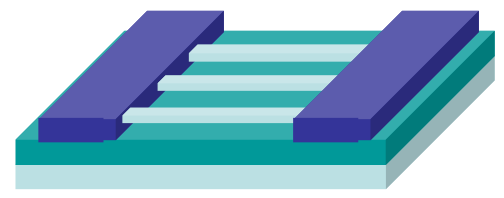

Bond control layer

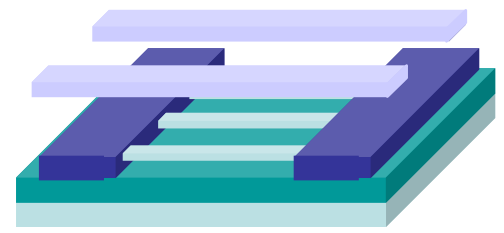

Remove multilayer structure and bond to Photonic Crystal

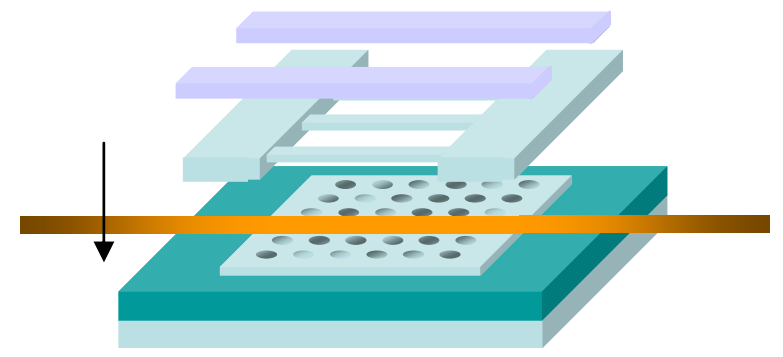

Figure 2: Overview of fabrication stages in nanoscale optofluidic integration technique. 
To complete the microfluidics engine an upper control layer which contains the control valves (see Unger et al. [31] for details of valve actuation in such devices) is bonded to the fluidic system. Briefly local flexure of the control layer when actuated by an external air pressure locally defines the pumps and valves which direct the flow on the bottom layer. The control layer is fabricated in the same fashion as the microfluidics layer however the final cast is made thicker ( 5mm) and with a 5:1 base:linker ratio. After both the fluidic and control layers have cured, the top side of the fluidic layer and bottom side of the PDMS layer are exposed to a short air plasma treatment, aligned, placed in conformal contact and finally baked at $80^{\circ} \mathrm{C}$ for two or more hours for final sealing. Figure 2 provides an overview of the fluidic fabrication procedure.

\subsection{Alignment and interfacing technique}

Alignment and interfacing the sub-wavelength features in the fluidic system with the sub-wavelength features in the photonic structures, complicated by the deformable nature of the fluidic system, is one of the most challenging aspects of this work. In general parallelity between the nanochannels and the photonic structure can be achieved through standard mechanical alignment techniques by ensuring that the alignment markers are separated by a sufficient distance. To align the nanochannels with the targeted row of holes in the y-direction (as defined in Figure 1) we vary the spacing between the array of photonic crystals by one half the diameter of the central row of holes. When interfaced with the regularly spaced array of nanochannels, this ensures that at least one nanochannel will be aligned with the central row of at least one photonic crystal. Using this technique the two systems were positioned using a standard mask aligner and then interfaced and bonded by placing them in conformal contact after a $7 \mathrm{~s}$ air plasma oxidation.

As will be demonstrated in the proceeding sub-section the "zebra-spacing" technique used here was successful at aligning the nanochannels with the targeted region of the photonic structure. In general however a more precise technique for both $\mathrm{x}$ and $\mathrm{y}$ directions is required to move towards twodimensional adaptable photonic structures. At present we are investigating the use of Moiré pattern techniques [32] for performing such alignment.

\subsection{Alignment, infusement and seal integrity tests}

After assembly, the nanochannels were initially infused with a $1 \%$ surfactant solution of Cetyltrimethylammonium bromide (CTAB) in DI water which served to reduce the surface tension at the air liquid interface and facilitate wetting of the nanochannel arrays. As mentioned above, the air permeable nature of the RTV fluidics was also likely instrumental in removing air bubbles which may otherwise have been trapped within the system. To directly examine the channel alignment and seal integrity at the nanoscale we conducted an experiment where we initially infused a highly concentrated CTAB solution (roughly $5 \%$ by mass) into the fluidic system and allowed the solvent to evaporate overnight. Figure 3 shows an SEM image of the CTAB residue after removal of the fluidics for both an aligned and misaligned case. The seal between the elastomer and photonic crystal is quite good within the crystal itself, however there appears to be some leakage around the outside of the device where the nanochannel first comes in contact with the photonic structure and must conform to the jump interface. As can be seen however the leakage remains confined outside the device and should not significantly affect the delivery.
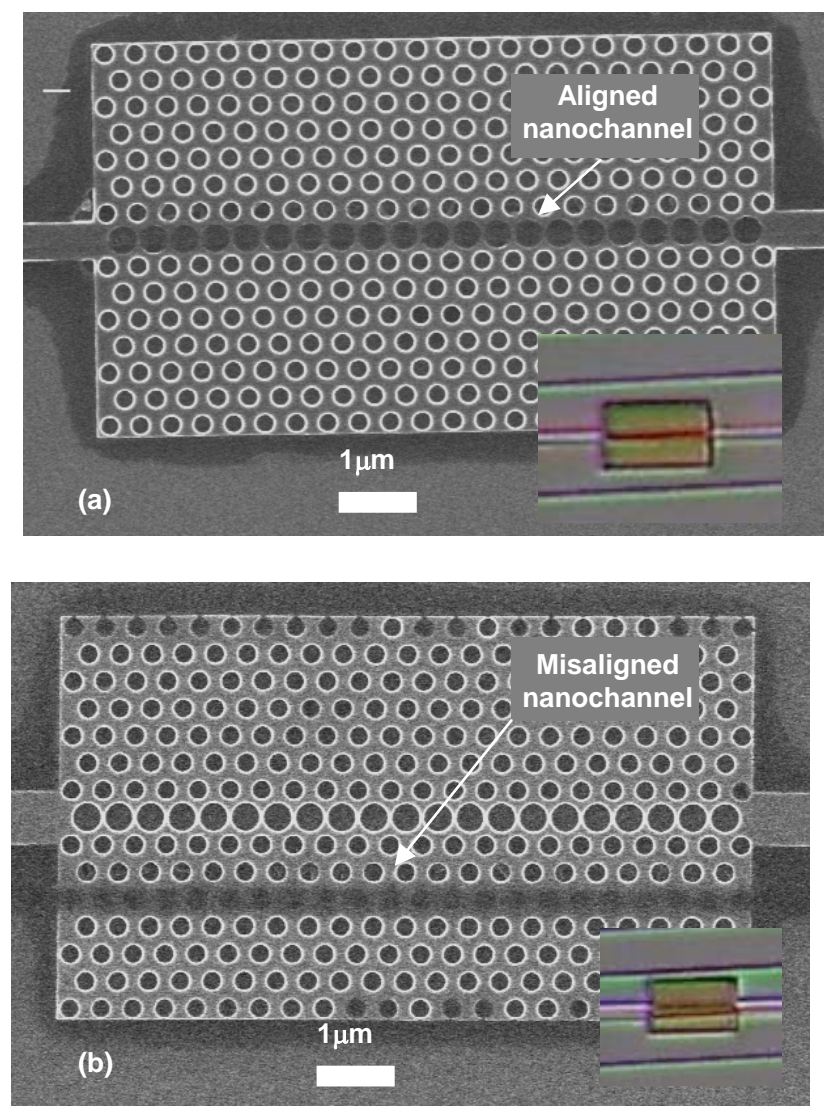

Figure 3. Demonstration of single row addressability for aligned nanochannel. (a) SEM image showing photonic crystal prior to integration with nanofluidics. Holes with increased radius define the region to be targeted fluidically. (b) SEM image of photonic crystal after removal of fluidics. Darkened regions show deposited CTAB after allowing the solvent to evaporate within the nanochannels, illustrating precise fluidic confinement within the targeted region of the photonic crystal.

As can be seen in both Figure 3a and 3b the infusement into the nanowell structures appears to be complete and there does not appear to be any nano- bubbles trapped within the wells. In this case the aspect ratio of the wells (height to diameter) is 0.74. Further studies are required in order to determine what 
the ultimate limit on the aspect ratio is for complete wetting of the structure.

\subsection{EXPERIMENTAL SECTION}

Figure 4 below illustrates the experimental layout used for these experiments. A typical experiment was conducted by first adhering the optofluidic structure to the $\mathrm{XZ}$ chip positioning arm (as shown in 4c) and inserting the fluidic and valve tubing (as shown in 4b). The fluidic system was then wetted using the technique described above. For this work we selected solutions of aqueous $\mathrm{CaCl}_{2}$ which ranged in refractive index from DI water, $n=1.33$, to $5 \mathrm{M}, \mathrm{n}=1.44$ as the fluids to modulate the refractive index in the photonic structure. While with greater far greater ranges in refractive index are available (see Erickson et al. [33]) the primary advantage of these solutions is the lack of an interface between the two fluids. The capillary pressure induced by the presence of an immiscible interface scales with 1/D and, as can be shown, at the channel scales used here this pressure becomes significantly higher than the pressure that can be applied to the system before failure of the fluidic seal.

Following mounting and connection of the fluidics tapered fiber lenses (Corning, Corning NY) were mounted in the fiber alignment stages as shown and connected to the HeNe fiber laser (Thorlabs) for preliminary alignment to the on-chip waveguides. Once the preliminary alignment was done the excitation fiber was connected to the tunable infrared laser (Agilent) and the collection fiber to the power meter. The position of both stages were then optimized to maximize the

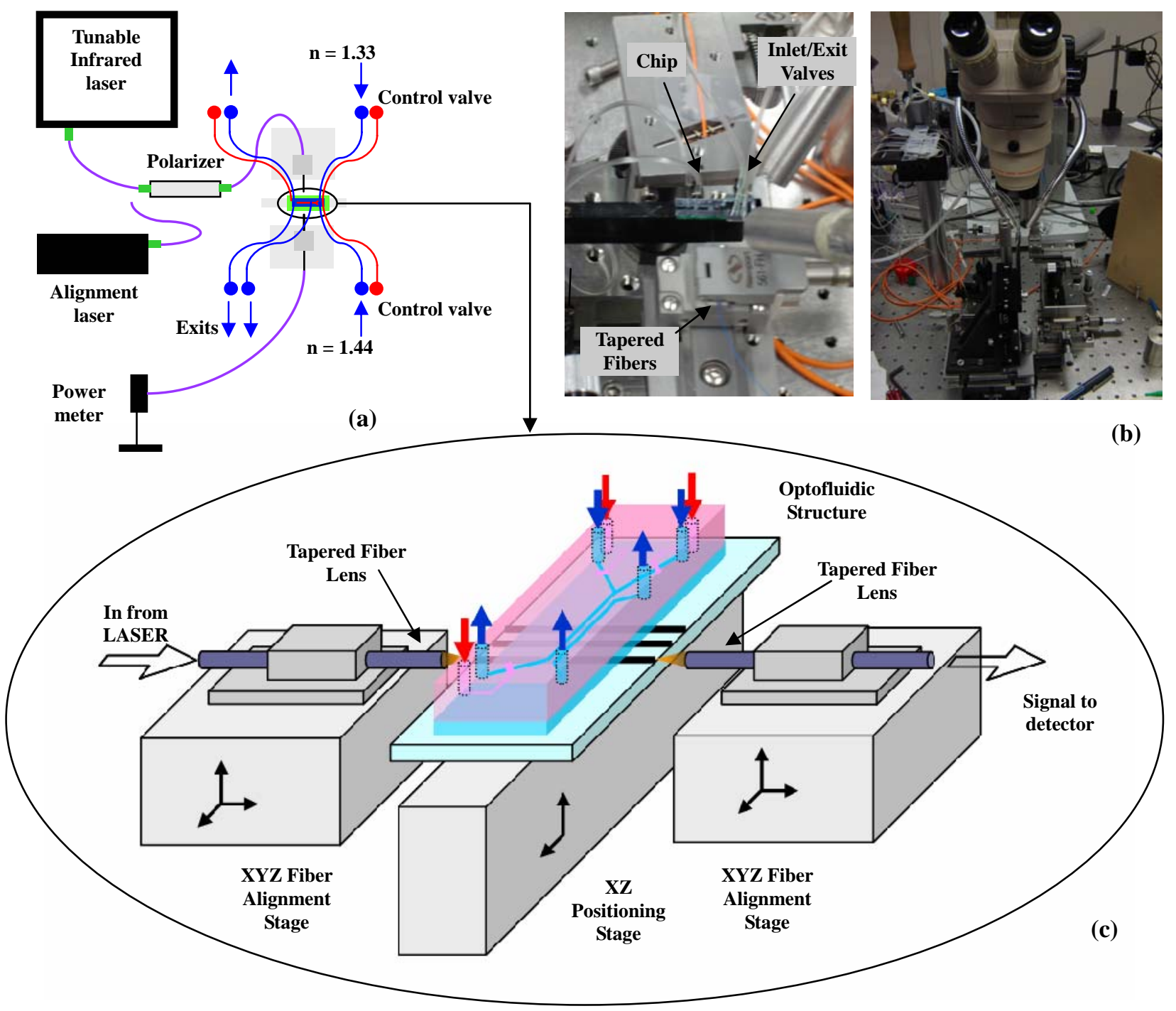

Figure 4. Nanofluidically tunable photonic crystals, experimental setup. (a) Layout of experimental setup showing fluidics, optics and positioning stages. (b) Photograph of setup (c) closeup image of the experimental apparatus. 
transmitted power through the photonic crystal. Once the system was aligned a scan was performed with the tunable laser over the available wavelength range $(1460 \mathrm{~nm}$ to $1580 \mathrm{~nm})$. To determine which of the photonic crystals was aligned with the nanofluidic structure the above procedure was repeated for each photonic crystal until the expected shift in the transmission spectrum was observed (as will be demonstrated in the following section). By performing an initial diagnostic inspection of the chip using an optical microscope we were able to reduce the number of photonic crystals which may be aligned to a handful of candidates, thereby eliminating the need to scan each system to find that which was correctly aligned. Once the system was aligned, spectrum were taken over the range of available wavelengths for both the DI solution and 5M $\mathrm{CaCl}_{2}$. Dynamic switching experiments were conducted between the two systems as will be outlined in the following section.

\subsection{NANOFLUIDIC TUNING OF PHOTONIC STRUCTURES}

Figure 5a shows the normalized quasi-TE mode transmission through the photonic crystal with a fluidically modulated waveguide (i.e. central row aligned with a nanofluidic channel) for DI water and $5 \mathrm{M} \mathrm{CaCl}_{2}$. The data presented has been smoothed to remove higher frequency Fabry-Perot resonances. The results show a shift in the peak transmission of the guided mode from $\mathrm{a} / \lambda=0.291$ to $\mathrm{a} / \lambda=0.289$ (corresponding to $\mathrm{a}$ $\Delta \lambda=15 \mathrm{~nm}$ ) when the lower index liquid is displaced by the higher index salt solution. The higher index solution effectively serves to decrease the size of the holes and shifts the guided mode towards the dielectric band of the regular crystal. As can be seen from Figure 5a increasing the index difference between the two fluids would provide a greater shift in the peak transmission of the guided mode, however it would not provide for a significantly higher extinction ratio. The dispersion diagram for photonic crystal geometry used here (see Figure 1). The $n=1$ line represents the guided mode which is introduced into the photonic crystal by increasing the radius of the holes in the targeted region. As the air was replaced with the higher refractive index liquids the frequency of the guided mode decreased as the optical radius of the holes became smaller. As can be seen the change the computed peak transmission wavelength matched well with that observed experimentally.

Dynamic modulation of the transmitted power is demonstrated in Figure $5 \mathrm{~b}$ at $\mathrm{a} / \lambda=0.291$ by fluidically switching between the DI water and $\mathrm{CaCl}_{2}$ solutions (shown schematically in Figure $5 c$ ). As can be seen extinction ratios on the order of $20 \mathrm{~dB}$ are obtained with a switching speed on the order of 10 s of seconds. The modulation speed is inherently limited by the viscous nature of the process. For pressure driven flows we can estimate the timescale for hydrodynamic switching by examining the time required to completely displace one liquid with another from a nanochannel given by $t_{\text {switch }}=12 \eta \mathrm{L}^{2} / \Delta P$ $\mathrm{D}_{\mathrm{h}}{ }^{2}$. The applied pressure, $\Delta \mathrm{P}$, for this case was between 15
$\mathrm{kPa}$ and $30 \mathrm{kPa}$ limited by the sealing stability between the PDMS and the photonic substrate. The channel hydraulic diameter, $\mathrm{D}_{\mathrm{h}}$, is fixed by the photonic geometry. The channel length, $\mathrm{L}$ was $400 \mu \mathrm{m}$ which could be reduced by at least an order of magnitude resulting in two order of magnitude decrease in the switching time however this is still in the millisecond range.
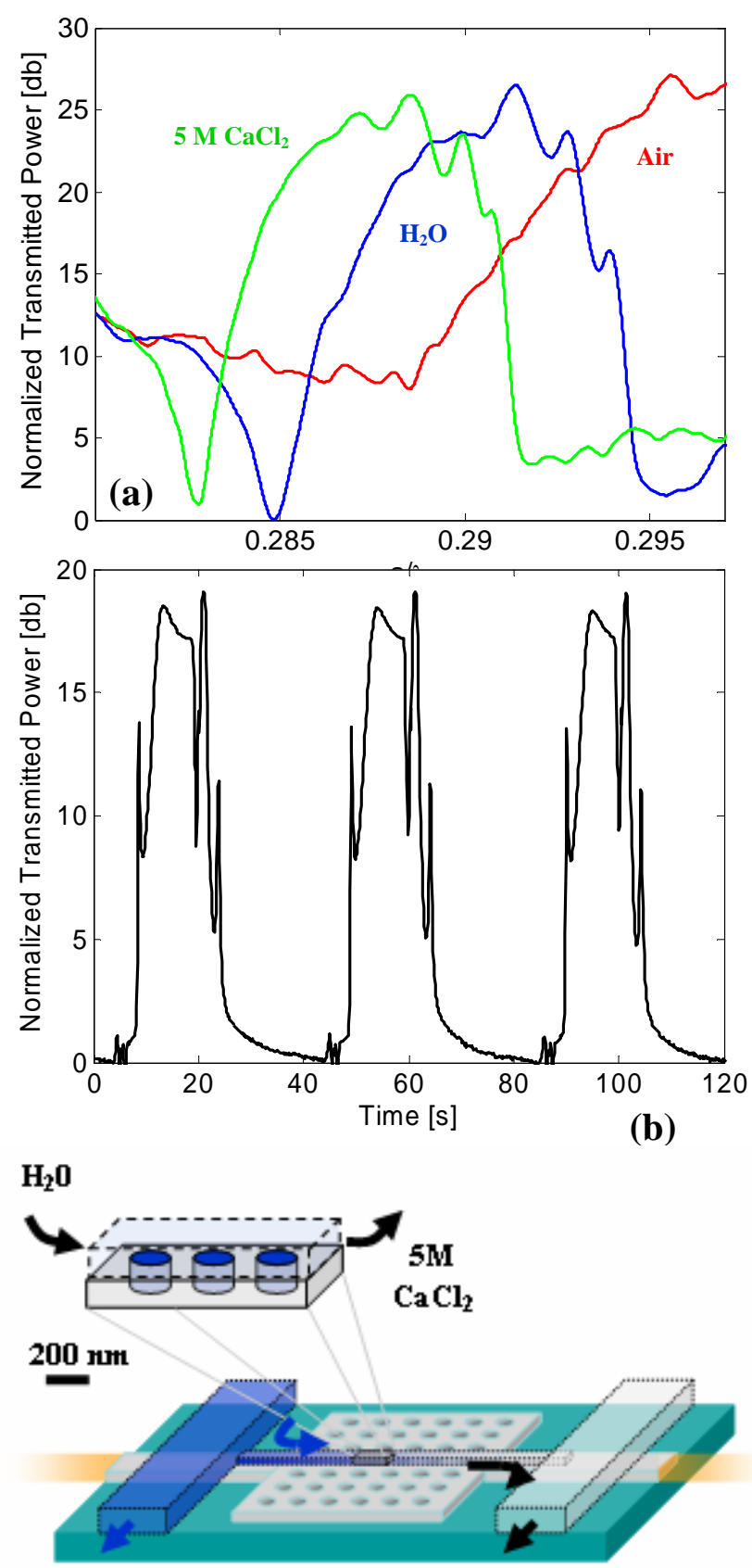

(c)

Figure 5: TE-like transmission through photonic crystal with aligned nanochannel. (a) Shift in transmitted power spectrum when aligned nanochannel filled with Air $(n=1), \mathrm{H}_{2} \mathrm{O}(n=$ 
1.33) and $5 \mathrm{M} \mathrm{CaCl}_{2}(n=1.44)$ (b) Dynamic switching at $\mathrm{a} / \lambda=$ 0.291. (c) Schematic showing dynamic displacement.

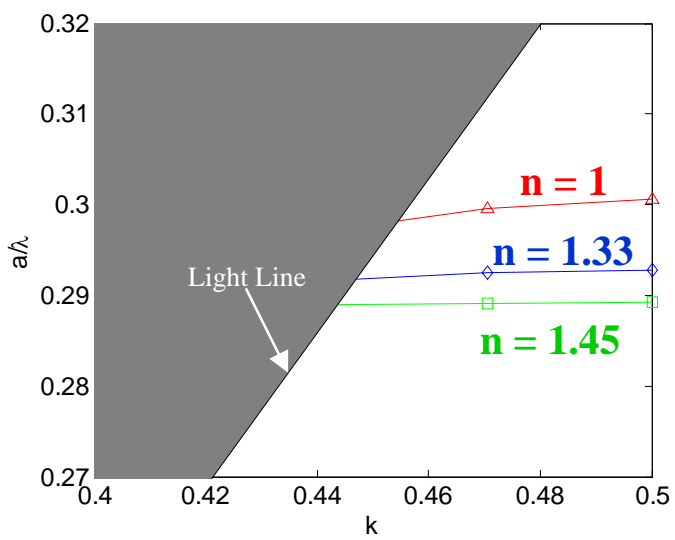

Figure 6: Dispersion diagram for TE-like modes in the for the $3 D$ photonic crystal geometry shown in Figure 1. Lines demonstrate the change in the location of the guided mode with modulation of the refractive index in the central row of holes.

\section{ACKNOWLEDGMENTS}

This work was carried out at the Center for Optofluidic Integration at the California Institute of Technology. Funding for the center is provided by the Defense Advanced Projects Agency under the University Photonics Research Centers Program.

\section{REFERENCES}

1. Joannopoulos, J., Meade, R., Winn, J., 1995, Photonic Crystals: Molding the Flow of Light. Princeton University Press, Princeton, New Jersey.

2. Johnson, S., Joannopoulos, J., 2002, Photonic Crystals: The Road from Theory to Practice. Kluwer Academic Publishers, Boston, Massachusetts.

3. Painter, O., Lee, R.K., Scherer, A., Yariv, A., O'Brien, J.D., Dapkus, P.D., Kim, I., 1999 Science, 284, 1819.

4. Almeida, V., Barrios, C., Panepucci, R., Lipson M., 2004, “All-optical switching on a silicon chip” Nature 431, 1081.

5. Xu, Q., Schmidt, B., Pradhan, S., Lipson, M., 2005, "Micrometer-scale silicon electro-optic modulator" Nature, 435, 325.

6. Park, W., Lee, J-B., 2004, Mechanically tunable photonic crystal structure. App. Phys. Lett. 85, 4845.
7. Chong, H., De La Rue, R., 2004, "Tuning of Photoic Crystal Waveguide by Thermooptic Effect." IEEE Photonics Tech. Lett. 161528.

8. Leonard, S., Mondia, J., van Driel, H., Toader, O., John, S., Busch, K., Birner, A., Gosele, U., Lehmann, V., 2005, "Tunable two-dimentional photonic crystals using liquidcrystal infiltration.” Phys. Rev. B 612389.

9. Kerbage, C., Windeler, R.S., Eggleton, B.J., Mach, P., Dolinski, M., Rogers, J., 2002, "Tunable devices based on dynamic positioning of micro-fluids in microstructured optical fiber.” Opt. Commun. 204, 179.

10. Mach, P., Dolinski, M., Baldwin, K., Rogers, J., Kerbage, C., Windeler, C., Eggleton, B., 2002, "Tunable microfluidic optical fiber.” Appl. Phys. Lett. 80, 4294.

11. Cattaneo, F., Baldwin, K., Yang, S., Krupenkine, T., Ramachandran, S., Rogers, J., 2003, "Digitally tunable microfluidic optical fiber devices.” J. MEMS 12, 907.

12. Raineri, F., Cojocaru, C., Raj, R., Monnier, P., Levenson, A., Seassal, C., Letartre, X., Viktorovitch, P., 2005, "Tuning a two-dimentional photonic crystal via optical carrier injection.” Opt Lett. 30, 64.

13. Tan, H., van Driel, H.M., Schweizer, S., Wehrspohn, R., Gosele, U., 2004, "Nonlinear optical tuning of a twodimentional silicon photonic crystal.” Phys. Rev. B 70 205110.

14. Iwamoto, S., Arakawa, Y., 2004, "Photonic Crystal with Advanced Micro/Nano-Structures: Quantum Dots and MEMS.” IEICE Trans. Electron. E87 343

15. Wild, B., Ferrini, R., Houdre, R., Mulot, M., Anand, S., Smith, C., 2004, "Temperature tuning of optical properties of planar photonic crystal microcavities.” Appl. Phys. Lett. 84846.

16. Loncar, M., Nedeljkovic, D., Doll, T., Vuckovic, J., Scherer, A., Pearsall, T., 2000, "Waveguiding in Planar Photonic Crystals” App Phys Lett. 771937.

17. Manz, A., Graber, N., Widmer, H., 1990, "Miniaturized total chemical analysis systems - A novel concept for chemical sensing” Sens. Actuators B 1244.

18. Manz, A., Harrison, D., Verpoorte, E., Fettinger, J., Paulus, A., Ludi, H., Widmer, H., 1992, "Planar chips technology for miniaturization and integration of separation techniques into monitoring systems - capillary electrophoresis on a chip” J. Chromatography, 593253. 
19. Harrison, D., Fluri, K., Seiler, K., Fan, Z., Effenhauser, C., Manz, A., 1993a, "Micromachining a miniaturized capillary electrophoresis-based chemical-analysis systems on a chip” Science 261, 895.

20. Petersen, K., 1982, "Silicon as a mechanical material" Proceedings of the IEEE $\mathbf{7 0} 420$.

21. Wise, K., Najafi, K., 1991, “Microfabrication techniques for integrated sensors and microsystems” Science 254, 1335.

22. Chabinyc, M., Chiu, D., McDonald, J., Stroock, A., Christian, J., Karger, A., Whitesides, G., 2001, “An integrated fluorescence detection system in poly(dimethylsiloxane) for microfluidic applications” Anal. Chem. 734491.

23. Duffy, D., McDonald, J., Schueller, O., Whitesides, G., 1998 "Rapid prototyping of microfluidic systems in poly(dimethylsiloxane)” Anal. Chem. 70, 4974.

24. Johnson, R, Badr,, I., Barrett, G., Lai, S., Lu, Y., Madou, M., Bachas, L., 2001, "Development of a fully integrated analysis system for ions based on ion-selective optodes and centrifugal microfluidics” Anal. Chem. 73, 3940.

25. Pugmire, D., Waddell, E., Haasch, R., Tarlov, M., Locascio, L., 2002, "Surface characterization of laserablated polymers used for microfluidics” Anal. Chem. 74, 871.

26. Becker H., Locascio, L.E., 2002, "Polymer microfluidic devices” Talanta, 56, 267.

27. deMello, A., 2002, "Microfluidics - DNA amplification moves on” Lab Chip, 2, 31N-36N.

28. Schueller, O., Brittain, S., Whitesides, G., 1999, "Fabrication of glassy carbon microstructures by soft lithography” Sensors and Actuators A, 72125.

29. Schueller, O., Duffy, D., Rogers, J., Brittain, S., Whitesides, G., 1998, "Reconfigurable diffraction gratings based on elastomeric microfluidic devices" Sensors Actuators A, 78, 149.

30. Thorsen, T., Maerkl, S., Quake, S., 2002, “Microfluidic large-scale integration” Science 298580.

31. Unger, M., Chou, H., Thorsen, T., Scherer, A., Quake S., 2000, "Monolithic microfabricated valves and pumps by multilayer soft lithograph” Science 288, 113.
32. King, M., Berry, D., 1972 "Photolithographic Mask Alignment using Moire Techniques" App. Optics, 112455.

33. Erickson, D., Heng, X., Li, Z., Rockwood, T., Emery, T., Zhang, Z., Scherer, A., Yang, C., Psaltis, D., "Optofluidics" SPIE Optics and Photonics Meeting, August 2005, San Diego. 